Historic, Archive Document

Do not assume content reflects current scientific knowledge, policies, or practices. 


\section{HAVE PREPARED FOR YOU}

Realizing the great interest in vegetable gardening this year and also realizing that but few are fortunate enough to possess a hotbed or cold frame and that many others may have forgotten to sow seeds early for things which should be started in March, I had prepared and have grown thousands upon thousands of vegetable plants ready to be set out on receipt.

It is surer than seeds and I dare say cheaper.

\section{BEET PLANTS}

Ready from now till June 1st Variety: Early Eclipse BRUSSEL SPROUTS PLANTS Ready after June 15th

Variety: Long Island improved

$$
\text { CABBAGE PLANTS }
$$

Ready now up to May 25th

Variety: Early Jersey Wakefield "Summer

Ready after May 25th

Varieties: Successian, Surehead Danish Ballhead, Drumhead Savoy, Red Drumhead

CAULIFLOWER PLANTS

Ready now up to May 25th

Variety: Early Snowball

\section{CELERY PLANTS}

Ready from May 1 st to June 25

Variety: Golden Self Blanching White Plume

Ready after June 25th

Variety: Winter Queen EGG PLANTS

Ready after May 1st

Variety: N. Y.Spineless LETTUCE PLANTS

Ready now up to May 25th Variety: Big Boston

PARSLEY PLANTS

Ready after June 1st

Variety: Dark Moss Curled

\begin{tabular}{|c|c|c|c|c|}
\hline \multicolumn{3}{|c|}{ via Mail prepaid } & \multicolumn{2}{|c|}{ via Express collect } \\
\hline per doz. & per 50 & per 100 & per 500 & per 1000 \\
\hline .25 & .75 & 1.25 & 3.25 & 5.50 \\
\hline .35 & .75 & 1.25 & 2.50 & 4.50 \\
\hline .35 & .85 & 1.35 & 5.00 & 8.00 \\
\hline .35 & .75 & 1.00 & 2.00 & 3.00 \\
\hline ' & & & & \\
\hline .40 & 1.00 & 1.60 & 5.00 & 8.75 \\
\hline .30 & .70 & 1.10 & 4.00 & 6.50 \\
\hline .25 & .70 & 1.10 & 2.50 & 3.50 \\
\hline .60 & 1.50 & 2.50 & 7.00 & 12.00 \\
\hline .25 & .75 & 1.20 & 3.00 & 5.00 \\
\hline $.30 y$ & .75 & 1.25 & 3.00 & 5.00 \\
\hline
\end{tabular}

$1917 ? \%$

PEPPER PLANTS

Ready after May 1st

Variety: Ruby King

SWEET POTATOE PLANTS

Ready after May 1 st

Variety: Yellow Nansemond

$$
\text { Red }
$$

"6

TOMATO PLANTS

Ready now up to June 10 th

Variety: Earliana, Bonnie Best, Stone, Jewel, Ponderosa,

\begin{tabular}{|c|c|c|c|c|}
\hline \multicolumn{3}{|c|}{ via Mail prepaid } & \multicolumn{2}{|c|}{ via Express collect } \\
\hline per doz. & per 50 & per 100 & per 500 & per 1000 \\
\hline .45 & 1.20 & 1.75 & 5.25 : & 9.00 \\
\hline .25 & .60 & 1.00 & 2.50 & 4.00 \\
\hline .40 & 1.00 & 1.50 & 5.00 & 7.50 \\
\hline
\end{tabular}
Matchless, Dwarf Stone.

I

\section{SPECIAL OFFER}

FULD'S HOME GARDEN COLLECTION consisting of

24 Early Cabbage Plants

36 Early Beet Plants

12 Early Cauliflower Plants

24 Early Celery Plants

Postpaid to any state in the Union for $\$ 2.50$

These plants are guaranteed to arrive in first class condition. Order Promptly.
24 Big Boston Lettuce Plants

12 Early Tomato Plants

12 Maincrop Tomato Plants

MAURICE FULD

Plantsman - Seedsman

1457 Broadway

NEW YORK 


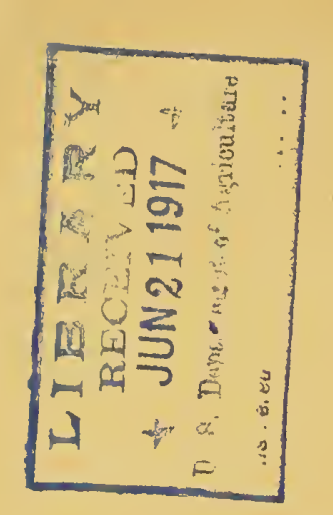

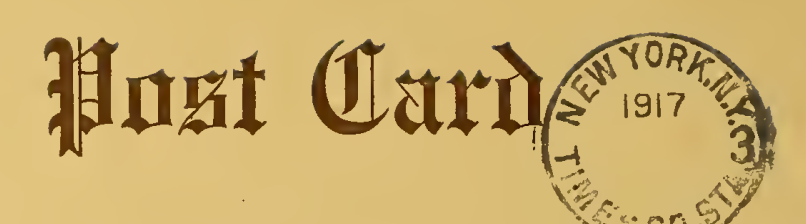

$\underline{\underline{\underline{E}}}$

Dn. E.E. Lereduff, 1825 Rorth Eapitolt. Eashingtor, OE. 